\title{
Revision Sistemática y Metaanálisis de Técnicas que Previenen el Dolor Lumbar
}

\author{
Prevention of Low Back Pain. \\ A Systematic Review \& Meta-analysis
}

\begin{abstract}
Paul Carey
'Southwest Center for Occupational \& Environmental Health, University of Texas Health Science Center at Houston, Houston, Texas.
\end{abstract}

\section{Resumen}

Este trabajo es un comentario del artículo: Steffens D, Maher CG, Pereira LSM, et al. Prevention of Low Back Pain: A Systematic Review and Meta-analysis. JAMA Intern Med. 2016;176(2):199-208. doi:10.1001/jamainternmed.2015.7431.

\section{Abstract}

This text is a commentary on the article: Steffens D, Maher CG, Pereira LSM, et al. Prevention of Low Back Pain: A Systematic Review and Meta-analysis. JAMA Intern Med. 2016;176(2):199-208. doi:10.1001/jamainternmed.2015.7431.

Sección coordinada por

Consol Serra (consol.serra@upf.edu) | Ma del Mar Seguí(mm.segui@ua.es)

Fechas · Dates

Recibido: 2020.04 .03

Publicado: 2020.04.15

\section{Correspondencia $\cdot$ Corresponding Author}

Paul Carey, MD MPH

Paul.carey@uth.tmc.edu 


\section{Resumen del artículo comentado}

Importancia: Las directrices actuales y las revisiones sistemáticas carecen de recomendaciones claras para la prevención del dolor lumbar.

Objetivo: Investigar la efectividad de las intervenciones para la prevención del dolor lumbar.

Fuentes de datos: MEDLINE, EMBASE, Physiotherapy Evidence Database Scale y Cochrane Central Register of Controlled Trials desde su inicio hasta el 22 de noviembre de 2014.

Seleccion de estudio: Ensayos clínicos aleatorios con estrategias de prevención para el dolor lumbar inespecífico.

Extracción de datos y síntesis: Dos analistas independientes extrajeron datos y evaluaron el riesgo de parcialidad. La escala de base de datos de evidencia de fisioterapia se utilizó para valorar el riesgo de parcialidad. El sistema para calificar la valoración de las recomendaciones, desarrollo y evaluación (GRADE) se utilizó para describir la calidad de la evidencia.

Principales resultados y medidas: La medida de resultado primaria era un episodio de dolor lumbar y la medida de resultado secundaria fue un episodio de ausencia laboral por enfermedad asociada con dolor lumbar. Calculamos los riesgos relativos (RR) y los intervalos de confianza (CI) del 95\% utilizando modelos de efectos aleatorios.

Resultados: La búsqueda bibliográfica identificó 6133 estudios potencialmente elegibles; de estos, 23 informes publicados (en 21 ensayos clínicos aleatorios diferentes, incluidos 30850 participantes únicos) cumplieron con los criterios de inclusión. Con los resultados presentados como riesgo relativo (intervalos de confianza del 95\%), hubo pruebas de calidad moderada de que el ejercicio combinado con la educación reduce el riesgo de un episodio de dolor lumbar (0,55 [0,41-0,74]) y pruebas de baja calidad sin efecto sobre la ausencia laboral por enfermedad $(0,74[0,44-1,26])$. La evidencia de baja a muy baja calidad sugirió que el ejercicio solo puede reducir el riesgo de un episodio de dolor lumbar $(0,65[0,50-0,86])$ y la ausencia laboral por enfermedad $(0,22[0,06-0,76])$. Solo para la educación, hubo evidencia de moderada a muy baja calidad de ningún efecto sobre el dolor lum$\operatorname{bar}(1,03[0,83-1,27])$ o la ausencia laboral por enfermedad $(0,87[0,47-1,60])$. Hubo evidencia de baja a muy baja calidad de que los cinturones de espalda no reducen el riesgo de episodios del dolor lumbar $(1,01[0,71-1,44])$ o ausencia laboral por enfermedad $(0,87[0,47-1,60])$. Hubo evidencia de baja calidad de ningún efecto protector de las plantillas de zapatos $(1,01[0,74-1,40])$.

Conclusión y relevancia: La evidencia actual sugiere que el ejercicio solo o en combinación con la educación es efectiva para prevenir el dolor lumbar. Otras intervenciones, como la educación sola, los cinturones de espalda y las plantillas de zapatos, no parecen a prevenir el dolor lumbar. Es incierto a causa de la baja calidad de la evidencia, si la educación, el entrenamiento o los ajustes ergonómicos previenen la ausencia laboral por enfermedad. 


\section{Comentario del artículo}

Steffens y colegas han identificado ensayos clínicos relevantes que han demostrado los beneficios del ejercicio solo y combinado con la educación para prevenir la incidencia del dolor lumbar, pero quedan preguntas fundamentales sobre este estudio. ¿El menor número de ensayos clínicos identificados y la calidad baja general del conjunto de evidencia justifica un metaanálisis por cada estrato de prevención? ¿Deberían los autores haber realizado una revisión sistemática descriptiva enfocada en los estudios individuales significativos de mejor calidad?

Aproximadamente el $80 \%$ de los ensayos clínicos identificados por Steffens y colegas presentan puntuaciones de PEDro inferiores a siete, lo que corresponde a una baja calidad metodológica. Los errores asociados con la calidad metodológica pueden implicar parcialidad estadística de sus conclusiones e influir en los resultados del metaanálisis ${ }^{(1)}$. Los ensayos clínicos aleatorios con falta de asignación oculta y cegamiento completo tienden a exagerar las estimaciones del efecto de prevención ${ }^{(1)}$. Las limitaciones metodológicas están presentes en la mayoría de los estratos de prevención y disminuyen el nivel de calidad general de la evidencia(2,3).

La preferencia por utilizar ensayos clínicos previamente calificados por parcialidad estadística dentro de la base de datos PEDro, que reúne solo 54000 referencias, reduce significativamente la capacidad para identificar un suficiente número de estudios clínicos e introduce parcialidades estadísticas asociadas con la selección de estudios ${ }^{(4)}$. En cuanto a la imprecisión estadística, la segunda categoría de la escala GRADE se refiere a un conjunto de ensayos clínicos agrupados en un estrato con menos de 400 participantes ${ }^{(2)}$. La escasez de ensayos clínicos degrada el componente de precisión estadística y baja aún más el nivel de la calidad general en aproximadamente el $60 \%$ de los metaanálisis de este estudio ${ }^{(2,3)}$ Asimismo, causa más sensibilidad a la heterogeneidad estadística, especialmente cuando existen efectos contradictorios dentro del mismo estrato de prevención ${ }^{(3)}$. Esto es evidente en el estrato de solo ejercicio a corto plazo para prevenir la incidencia del dolor lumbar. Los ensayos clínicos agrupados en este estrato contienen un estudio con efecto de prevención contradictorio de los otros, inferido por Helewa y colegas ${ }^{(5)}$. Esta contradicción dentro del este mismo estrato, resulta en niveles de heterogeneidad estadística moderado y alto. Steffens y colegas reportaron que este estrato contenía $I^{2}=67,9 \%$, superando el límite de la heterogeneidad estadística de $I^{2}>50,0 \%{ }^{(4)}$. Con respecto a la inconsistencia de los resultados, el tercer componente de la escala GRADE se refiere a cuando se sobrepasan los límites prescritos de la heterogeneidad estadística. El alto nivel de heterogeneidad en el estrato de solo ejercicio a corto plazo, reduce aún más el nivel de la calidad general utilizado en este metaanálisisis ${ }^{(2,3)}$.

El estrato de prevención con ejercicio a corto plazo para prevenir la incidencia del dolor lumbar incluyó algunos estudios clínicos relevantes de Sihawong y colegas ${ }^{(6)}$. Este estudio concluyó una baja incidencia del dolor lumbar estadísticamente significativa con el ejercicio a corto plazo. Dentro de la misma estratificación incluyó estudios clínicos de Helewa y Warming, que compararon las intervenciones educativas solas y en combinación con el ejercicio ${ }^{(5,7)}$. El estrato de prevención 
del dolor lumbar con ejercicio a largo plazo también incluyó datos del estudio Helewa y colegas en el metaanálisis de un efecto sumativo(5). Una gran preocupación de los dos metaanálisis en los estratos de solo ejercicio a corto y largo plazo es la confusión estadística dada por los estudios de Helewa y Warming ${ }^{(5,7)}$. Ambos ensayos clínicos compararon prevenciones de solo educación y educación con ejercicio, pero no estudiaron el ejercicio solo ${ }^{(5,7)}$. Estas inconsistencias confunden el cálculo del efecto sumativo. Los metaanálisis consisten en datos influenciados por parcialidad estática basada en los dos estudios clínicos mal asignados y proyectan más dudas sobre las conclusiones derivadas ${ }^{(8)}$.

El metaanálisis de la prevención con ejercicio y educación a corto plazo demuestra una magnitud del efecto sumativo intermedio. El resultado respalda los ensayos clínicos individuales que demuestran una reducción en la incidencia del dolor lumbar mediante evidencia de calidad moderada. El metaanálisis de ejercicio y educación a largo plazo demuestra una magnitud del efecto sumativo menor que a corto plazo. El resultado también respalda los ensayos clínicos individuales que demuestran una disminución en la incidencia del dolor lumbar mediante evidencia de baja calidad. Steffens y colegas reportaron una diferencia numérica de los efectos sumativos de ejercicio y educación a corto y largo plazo obtenidos por metaanálisis. Los autores deberían tener precaución al reportar diferencias cuantitativas por la falta de precisión asociada con la evidencia de baja calidad utilizada en los metaanálisis y que pone en duda sus conclusiones ${ }^{(9)}$.

Los ensayos clínicos individuales sobre intervenciones como uso de plantillas, cinturones y educación sola, no proporcionaron cambios estadísticamente significativos o discernibles para disminuir el riesgo de la incidencia o ausencia laboral por enfermedad relacionada con el dolor lumbar. Conjuntamente, los metaanálisis de estos tipos de prevención reflejaron la naturaleza estadística inconclusa de la evidencia general, por lo que no derivaron conclusiones claras.

En resumen, el bajo número de ensayos clínicos, la parcialidad estadística, la falta de precisión metodológica y las inconsistencias en los datos clínicos de prevención dentro del mismo estrato reflejaron una baja calidad general de la evidencia según lo determinado por la escala $\operatorname{GRADE}^{(2,8,9)}$. Una revisión cualitativa descriptiva que destacara los ensayos clínicos significativos hubiera sido más apropiada debido a la falta de buena evidencia clínica ${ }^{(10)}$. Una revisión sistemática podría haber eludido las demandas estadísticas de un metaanálisis, lo que hubiera proporcionado un contexto valioso de los limitados ensayos clínicos de buena calidad.

\section{Referencias}

1. Wood L, Egger M, Gluud LL, Schulz KF, Jüni P, Altman DG et al. Empirical evidence of bias in treatment effect estimates in controlled trials with different interventions and outcomes: meta-epidemiological study. BMJ. 2008;336:601-5.

2. Atkins D, Eccles M, Flottorp S, Guyatt GH, Henry D, Hill S et al. Systems for grading the quality of evidence and the strength of recommendations I: Critical apprai- 
sal of existing approaches The GRADE Working Group. BMC Health Serv Res. [edición electronica] 2004 Dec 22 [citado el 3 Mar 2020];4:38 [aprox. 7 p]. Disponible en: http://www.biomedcentral.com/1472-6963/4/38.

3. Mikolajewicz N, Komarova SV. Meta-Analytic Methodology for Basic Research: A Practical Guide. Front Physiol. [edición electronica] 2019 Mar 27 [citado el 3 Mar 2020];10:203 [aprox. 20 p]. Disponible en: https://www.frontiersin.org/articles/10.3389/fphys.2019.00203/full.

4. Steffens D, Maher CG, Pereira LSM, Stevens ML, Oliveira VC, Chapple M et al. Prevention of Low Back Pain: A Systematic Review and Meta-analysis. JAMA Intern Med. 2016;176:199-208.

5. Helewa A, Goldsmith CH, Lee P, Smythe HA, Forwell L. Does strengthening the abdominal muscles prevent low back pain - a randomized controlled trial. J Rheumatol. 1999;26:1808-1815.

6. Sihawong R, Janwantanakul P, Jiamjarasrangsi W. A prospective, cluster-randomized controlled trial of exercise program to prevent low back pain in office workers. Eur Spine J. 2014;23:786-793.

7. Warming S, Ebbehøj NE, Wiese N, Larsen LH, Duckert J, Tønnesen H. Little effect of transfer technique instruction and physical fitness training in reducing low back pain among nurses: a cluster randomised intervention study. Ergonomics. 2008;5:1530-1548.

8. Mathes T, Kuss O. A comparison of methods for meta-analysis of a small number of studies with binary outcomes. Res Syn Meth. 2018;9:366-381.

9. Melsen WG, Bootsma MCJ, Rovers MM, Bonten MJM. The effects of clinical and statistical heterogeneity on the predictive values of results from meta-analyses. Clinical Microbiology and Infection. 2014;20:123-129.

10. Egger M, Jüni P, Bartlett $C$, Holenstein F, Sterne J. How important are comprehensive literature searches and the assessment of trial quality in systematic reviews? Empirical study. Health Technol Assess (Rockv). 2003;7:1-76. 